\title{
Obscure gastrointestinal bleeding secondary to gastroduodenal pseudoaneurysm
}

\author{
Sanket Thakore MD, Omar N Nadhem MD, Essam Nakhla MD, Steven Urban MD
}

\begin{abstract}
Among the uncommon etiologies of gastrointestinal bleeding, gastroduodenal artery pseudoaneurysm is a rare condition that complicates acute recurrent pancreatitis, trauma, endoscopic retrograde cholangiopancreatography, cholecystectomy, and other conditions. It is a life threatening condition that carries a mortality rate of $40 \%$ if not treated. We report a case of a 51-year-old man with a history of recurrent acute pancreatitis who presented with repeated episodes of upper gastrointestinal bleeding from an uncertain source that required multiple admissions and blood transfusion. After thorough evaluation, the source was found to be a gastroduodenal artery pseudoaneurysm secondary to recurrent idiopathic acute pancreatitis that was successfully treated with catheter embolization.
\end{abstract}

Key words: pseudoaneurysm, gastroduodenal artery pseudoaneurysm, gastrointestinal bleeding, visceral angiography.

\section{INTRODUCTION}

Upper gastrointestinal bleeding is one of the leading causes of admission to the emergency department. ${ }^{1}$ Hemorrhage from the ampulla of Vater via the pancreatic duct, known as hemosuccus pancreaticus (HP), is a rare cause of intermittent upper gastrointestinal bleeding. ${ }^{2}$ Hemosuccus pancreaticus usually occurs as a complication of acute or chronic pancreatitis. During an episode of acute pancreatitis, HP occurs after necrosis of arterial walls or rupture of pseudoaneurysms originating from the enzymatic destruction of the arterial walls. When the involved ar-

Corresponding author: Sanket R. Thakore MD Contact Information: Sanket.Thakore@ttuhsc.edu DOI: 10.12746/swrccc2015.0312.155 tery or pseudoaneurysm communicates with the pancreatic duct, blood may enter the duct and empty from the ampulla into the duodenum. ${ }^{3}$ Gastroduodenal artery (GDA) pseudoaneurysms comprise only $1.5 \%$ of all visceral artery aneurysms. Although sometimes asymptomatic ( $7.5 \%$ of cases), GDA pseudoaneurysms usually present with pain in the upper abdomen, gastrointestinal or intraperitoneal bleeding, or anemia. In the management of pseudoaneurysms, early diagnosis is essential, given the high mortality of untreated bleeding. Endoscopy is useful to exclude other sources of hemorrhage, as visualization of the bleeding from the papilla is a rare event. ${ }^{6}$ Ultrasound with Doppler or CT scan may aid in the diagnosis. Visceral angiography is the gold standard diagnostic method. ${ }^{7}$ Percutaneous angiographic embolization is the treatment of choice for hemodynamically stable 
patients because it can achieve definitive hemostasis. Surgery should be reserved for hemodynamically unstable patients or for embolization failure. ${ }^{8}$ We report a case of patient with recurrent idiopathic acute pancreatitis who presented with repeated episodes of upper gastrointestinal bleeding due to a leaking GDA pseudoaneurysm.

\section{CASE}

A 51-year-old man with a past medical history of recurrent episodes of idiopathic pancreatitis over the last 2 years and past a surgical history of cholecystectomy presented to the hospital with epigastric pain, nausea, and vomiting of 2 days duration.

At admission, his hemoglobin level was 13.2 $\mathrm{gm} / \mathrm{dl}$, amylase 247 unit/L (normal 36-128), and lipase 293 unit/L (normal 10-57). A computed tomography scan of his abdomen with IV contrast showed peripancreatic inflammation and a hypodense area within the pancreatic head (Figure 1). On the second day, the patient had three melanotic stools (estimated blood loss $750 \mathrm{cc}$ ), and his hemoglobin level dropped to $8.7 \mathrm{gm} / \mathrm{dL}$. Colonoscopy and EGD failed to reveal any source of bleeding. The patient's condition then improved with minimal abdominal pain, diminishing

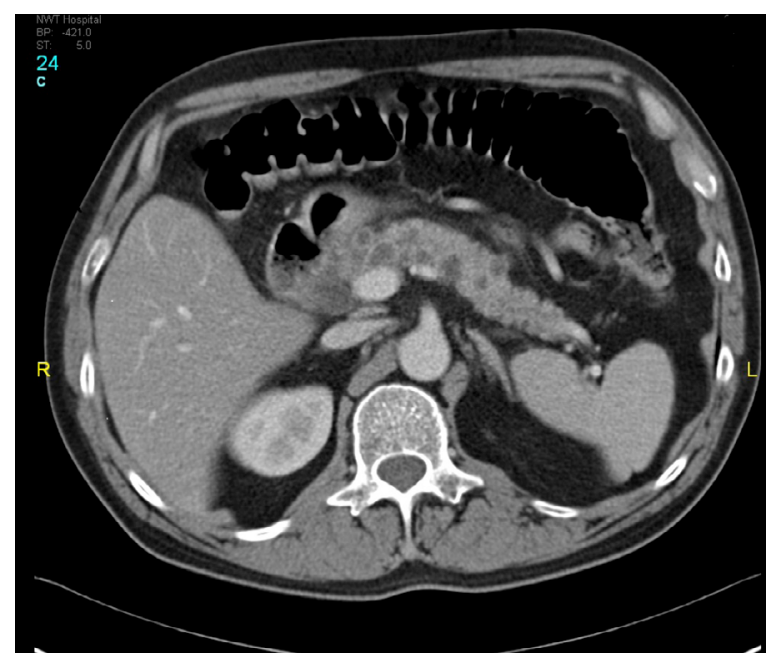

Figure 1: Acute pancreatitis with peripancreatic inflammation. Nonspecific hypodense areas within the pancreatic head and multiple pancreatic cystic lesions. pancreatic enzymes, no more bleeding episodes, and stable hemoglobin level. No blood transfusion was required, and he was discharged to follow-up with the gastroenterologist.

A few days later, the patient developed another episode of severe abdominal pain and was admitted with acute pancreatitis. His amylase and lipase levels were 425 and 618 unit/L, respectively, and his hemoglobin was $9.5 \mathrm{gm} / \mathrm{dL}$. Shortly after admission, we witnessed an episode of hematemesis (about 250 cc of fresh blood). Since he was actively bleeding, a tagged RBC scan was done but failed to identify the source of bleeding. His hemoglobin dropped to 6.5 $\mathrm{gm} / \mathrm{dl}$, and he received 3 units of packed RBCs. EGD and colonoscopy were repeated and again failed to identify any pathology. We then proceeded with CT angiography that showed no vascular abnormality in the abdomen that could explain the ongoing bleeding. To further pinpoint the diagnosis, we obtained capsule endoscopy which showed no source of bleeding below the ligament of Treitz.

Endoscopic ultrasound was subsequently done with fine needle aspiration of the hypodense lesion of pancreatic head revealing necrosis (i.e, necrotizing pancreatitis). Computed tomography enterography was also done with negative results. The patient then underwent small bowel enteroscopy with fluoroscopy looking for any source of bleeding into the small bowel. As the endoscope was advanced through the duodenum, fresh blood was seen coming from the ampulla of Vater with a clot in the ampulla.

The gastroenterologist stopped the procedure, and the patient was immediately transferred to the interventional radiology department for catheter arteriogram of the celiac and superior mesenteric arteries. This demonstrated a pseudoaneurysm originating from the gastroduodenal artery (Figure 2). Catheter embolization was performed with multiple coils placed proximal and distal to the pseudoaneurysm (Figure 3). Post-embolization images demonstrate no flow through the gastroduodenal artery and no enhancement of the pseudoaneurysm (Figure 4). The patient had no further episodes of gastrointestinal bleeding 
and was discharged home. He was seen later on in the gastroenterology clinic with complete resolution of his symptoms.

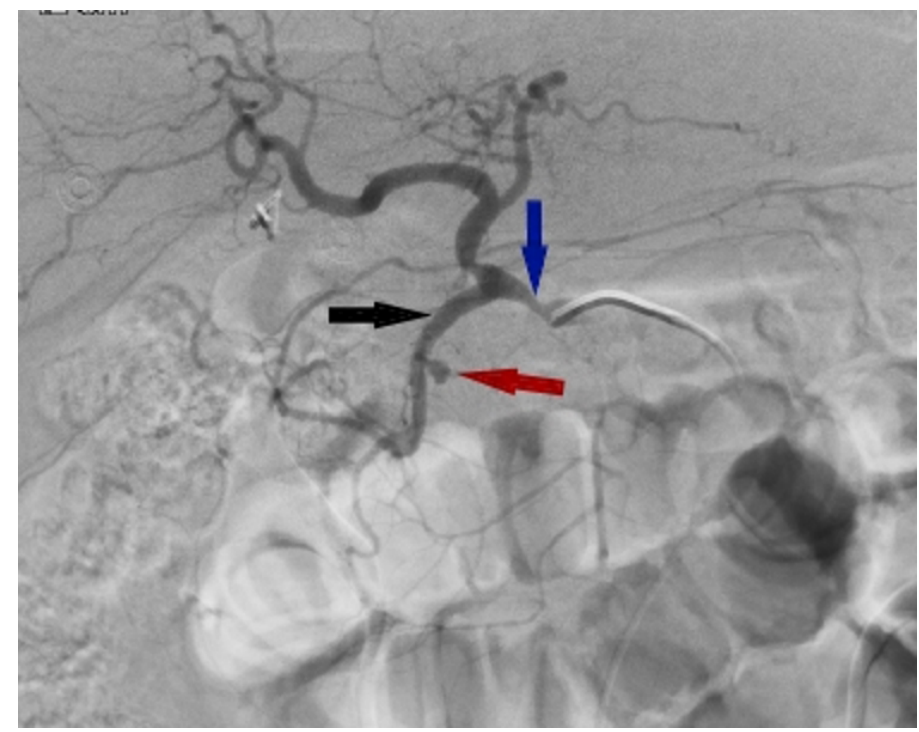

Figure 2: Digital Subtraction Arteriogram demonstrated a pseudoaneurysm originating from the gastroduodenal artery [GDA]. Black arrow shows GDA. Red arrow shows pseudoaneurysm originating from GDA. Blue arrow shows Common Hepatic artery.

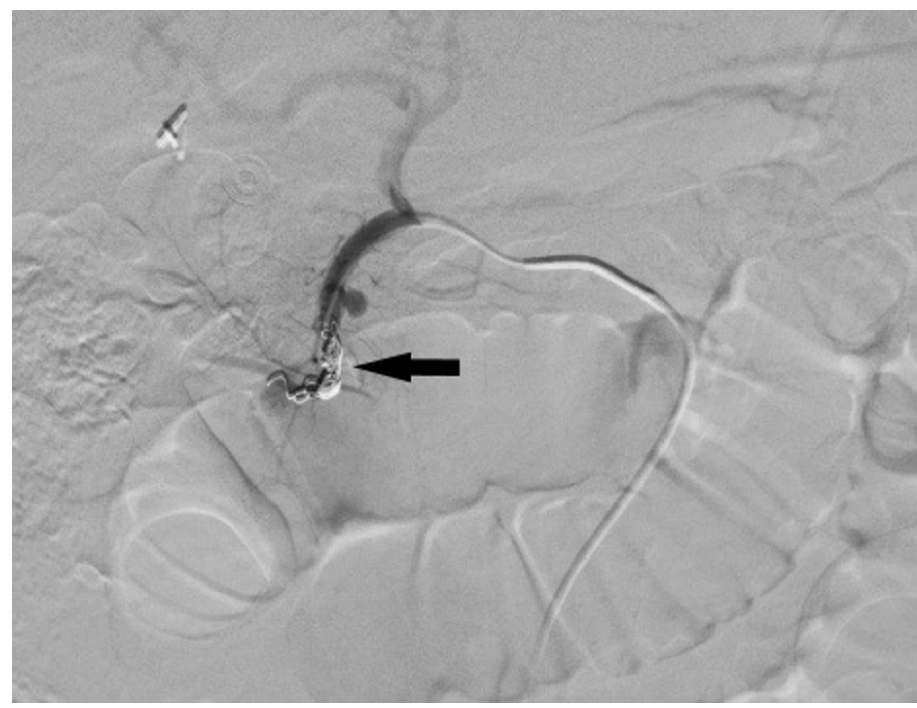

Figure 3: It shows coil embolization of GDA. Black arrow shows coil placed distally to the pseudoaneurysm.

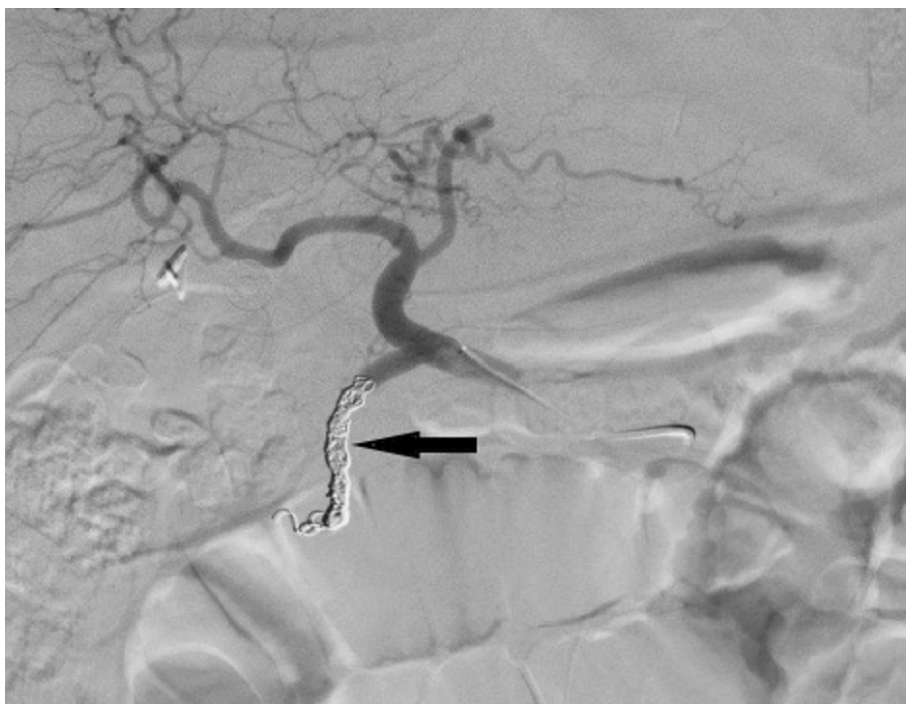

Figure 4: Digital Subtraction Arteriogram confirms absence of flow through the gastroduodenal artery and the pseudoaneurysm [black arrow] post coil embolization.

\section{Discussion}

Upper gastrointestinal bleeding is a leading cause of admission to the emergency department. Hemorrhage from the papilla of Vater via the pancreatic duct, known as hemosuccus pancreaticus, is a rare cause of intermittent upper gastrointestinal bleeding that usually develops from complications of pancreatitis. ${ }^{2,7}$ Visceral artery pseudoaneurysms are responsible for $20 \%$ of hemosuccus pancreaticus cases, and gastroduodenal artery pseudoaneurysms are among the rarest forms of visceral artery pseudoaneurysm $(<2 \%){ }^{9}$ Pseudoaneurysms are primarily a condition of middle age and most commonly found between 50 and 58 years of age. The male/female ratio is $4.5: 1$, and mean size is $3.6 \mathrm{~cm}$.

GDA pseudoaneurysms are the result of vessels exposed to proteolytic pancreatic enzymes or erosion of a pancreatic pseudocyst into an adjacent artery, which leads to pseudoaneurysm formation or bleeding. ${ }^{8,10}$ Once a GDA pseudoaneurysm ruptures, the patient faces a life threatening condition that rapidly leads to death in $40 \%$ of cases. ${ }^{4}$ Patients can present with nonspecific symptoms, such as nausea, 
vomiting, and increased abdominal pain, but they may present with more serious symptoms, including melena, hematochezia, or hematemesis. ${ }^{5}$ The presence of a pulsatile abdominal mass with or without a bruit on auscultation can be the sole warning sign and should raise the suspicion of a GDA aneurysm with a prompt diagnostic work-up to prevent a catastrophic outcome. ${ }^{4}$ Bleeding due to the pseudoaneurysm commonly occurs in the bowel followed by the peritoneal cavity, pancreatic duct, and biliary tree. ${ }^{5}$ Endoscopic diagnosis of hemosuccus pancreaticus is made when blood is seen coming from the ampulla of Vater. This finding is uncommon because bleeding is intermittent. $^{7}$ The intermittent nature of the bleeding is thought to occur because increased pressure in the pancreatic duct eventually seals and leads to thrombosis of its communication with the pseudoaneurysm. However, once the pancreatic duct decompresses and the clot lyses, the cycle can repeat itself. Bleeding events can occur infrequently over a period of months to years or can occur in rapid succession.

The gold standard diagnostic test is visceral angiography; it serves both diagnostic and therapeutic purposes by delineating the arterial anatomy and allowing therapeutic intervention. ${ }^{4}$ Recently, embolization, with or without endoscopic stent placement, has been considered the first therapeutic method for ruptured pseudoaneurysm. Success rates range $78 \%-100 \%$ in peripancreatic pseudoaneurysms secondary to pancreatitis. ${ }^{5,11}$ Angioembolization is considered much less invasive than surgery. The procedure can be completed quickly and is comfortable for the patient. ${ }^{5}$ Failure of catheter embolization may result from inability to isolate the bleeding vessel, incomplete arterial occlusion, or misidentification of the bleeding vessel. ${ }^{7}$ When coil embolization fails to control the bleeding, a surgical approach is usually necessary. ${ }^{5}$ The mortality rate is high even if surgery is performed. $^{11}$

In conclusion, hemosuccus pancreaticus due to rupture GDA pseudoaneurysm, especially in patient with recurrent acute or chronic pancreatitis, should be considered in the differential diagnosis of intermittent upper gastrointestinal bleeding of obscure source. In our case, active bleeding from the ampulla of Vater at the time of endoscopy led to immediate catheter angiography and successful ablation of the pseudoaneurysm.

\begin{abstract}
TTUHSC in Amarillo, TX.
Received: 06/15/2015

Accepted: 09/10/2015

Reviewers: Tinsay Woreta MD

Published electronically: 10/15/2015

Conflict of Interest Disclosures: none
\end{abstract}

Author Affiliation: Sanket Thakore, Omar N Nadhem, and Essam Nakhla are residents in Internal Medicine at Texas Tech University Health Sciences Center in Amarillo, TX. Steven Urban is a faculty member in Internal Medicine at

\section{REFERENCES}

1. Elazary R, Abu-Gazala M, Schlager A, Shussman N, Rivkind AI. Therapeutic angiography for giant bleeding gastro-duodenal artery pseudoaneurysm. World J Gastroenterol 2010; 16:16701672.

2. Toyoki Y, Hakamada K, Narumi S, Nara M, Ishido K. Hemosuccus pancreaticus: problems and pitfalls in diagnosis and treatment. World J Gastroenterol 2008; 14:2776-2779.

3. Han B, Song Z, Sun B. Hemosuccus pancreaticus: a rare cause of gastrointestinal bleeding. Hepatobiliary Pancreat Dis Int 2012; 11:479-488.

4. Habib N, Hassan S, Abdou R, Torbey E, Alkaied H, Maniatis T, Chalhoub M, Harris K. Gastroduodenal artery aneurysm, diagnosis, clinical presentation and management: a concise review. Ann Surg Innov Res 2013; 7:1750-1164.

5. Patel SB, Shah SR, Shah SS, Kumar NA. Case report: Pseudoaneurysm from gastroduodenal artery associated with chronic pancreatitis; an unusual complication. Indian J Radiol Imaging 2003; 13:311-313.

6. Elton E, Howell DA, Amberson SM. Combined angiographic and endoscopic management of bleeding pancreatic pseudoaneurysms. Gastrointestinal Endoscopy 1997; 46:544-549. 
7. Bohl JL, Dossett LA, Grau AM. Gastroduodenal artery pseudoaneurysm associated with hemosuccus pancreaticus and obstructive jaundice. J Gastrointest Surg 2007; 11:1752-1754.

8. Germanos S, Soonawalla Z, Stratopoulos C. Pseudoaneurysm of the gastroduodenal artery in chronic pancreatitis. $\mathrm{J} \mathrm{Am} \mathrm{Coll}$ Surg 2009; 208:316.

9. Saqib NU, DuBose J, Martin GH, Charlton-Ouw KM, Coogan SM, Estrera AL, Safi HJ. Coil embolization of a ruptured gastroduodenal artery pseudoaneurysm presenting with hemosuccus pancreaticus. J Vasc Surg 2013; 58:1737-1738.

10. Sah PL, Rauniyar RK, Gupta RK, Dhungel K, Ahmad K. Spontaneous rupture of pseudoaneurysm of gastroduodenal artery complicating pancreatitis: MDCT diagnosis. NJR 2011; $1: 30-32$.

11. Yamakado K, Nakatsuka A, Tanaka N, Takano K, Matsumura $\mathrm{K}$. Transcatheter arterial embolization of ruptured pseudoaneurysms with coils and n-butyl cyanoacrylate. JVIR 2000; 11:6672. 